\title{
368 CONSISTENT PATTERN OF IMMUNE ACTIVATION INDUCED BY ONCOLYTIC ADENOVIRUS ONCOS-102 ACROSS DIVERSE TYPES OF SOLID TUMORS
}

${ }^{1}$ Lukasz Kuryk, ${ }^{1}$ Anne-Sophie Moller, ${ }^{1}$ Sandeep Kumar, ${ }^{2}$ Alexander Shoushtari, ${ }^{3}$ Luis Paz Ares, ${ }^{1}$ Magnus Jaderberg, 'Erik Digman Wiklund, 'Victor Levitsky*. ${ }^{1}$ Targovax ASA, Oslo, Norway; ${ }^{2}$ Memorial Sloan Kettering Cancer Center, New York, NY, USA; ${ }^{3}$ Hospital Universitario 12 Octubre, Madrid, Spain

Background Solid tumors exhibit highly variable compositions of immune infiltrates. Therapeutic compounds driving uniform remodeling of tumor microenvironment (TME) across tumor types may improve the efficacy of cancer immunotherapy. ONCOS-102, a granulocyte-macrophage colony stimulating factor (GM-CSF)-expressing oncolytic adenovirus (Ad5/3-D24GMCSF), was tested for its safety, therapeutic efficacy and capacity to remodel TME in recently completed phase I/II clinical studies in anti-PD-1 refractory melanoma (NCT03003676) and malignant pleural mesothelioma (MPM) (NCT02879669).

Methods Biopsies were obtained from tumor lesions of patients treated with intra-tumoral injections of ONCOS-102 in combination with chemotherapy or pembrolizumab for MPM and melanoma, respectively. Tumor immune infiltrates were analyzed by immunohistology using several antibody panels. On-treatment biopsies were compared to paired baseline samples as wells as to samples from control patients treated with chemotherapy alone in the case of MPM. Gene expression data obtained by next generation RNA sequencing were used to complement the immunohistology analysis and all results were correlated to clinical outcomes.

Results Comparative TME analysis of anti-PD-1 refractory melanoma and MPM tumors revealed noticeably lower baseline T-cell infiltration in mesothelioma. Thus, fractions of CD8 + T-cells were significantly below $10 \%$ in $80 \%$ of MPM biopsies while approaching or exceeding this level in $60 \%$ of melanoma baseline samples. Comparison of tumor biopsies obtained at baseline or on-treatment, demonstrated increased infiltration by both $\mathrm{CD} 4+$ and $\mathrm{CD} 8+\mathrm{T}$-cells in large proportions of melanoma (CD4+: 13/20 (65\%); CD8+: 16/19 (84\%) and MPM (CD4+: 10/15 (67\%); CD8+: 9/15 (60\%) tumor lesions in response to ONCOS-102. Frequencies of cytotoxic T-cells with high granzyme-B expression also increased in response to the treatment in both tumor types, in particular when assessed as percentage of total CD8 + T-cells. Other observed changes induced by ONCOS-102 in samples taken from CR, PR and SD patients with MPM or melanoma included increased CD8/Treg ratio and modulation of PD-L1 expression. Biological and clinical importance of these findings was further supported by correlation between modulation of several subsets of genes related to the process of T-cell activation, such as cytotoxic granule components and co-stimulatory molecules, and clinical response to ONCOS-102 in melanoma and both tumor response and overall survival in MPM patients.

Conclusions ONCOS-102 drives pro-inflammatory modulation of immune TME across tumor types of different origins, anatomical locations and immunological baseline characteristics. Our data support potential of ONCOS-102 to serve as a potent immune sensitizing agent in combination therapies with various classes of immunomodulatory compounds and chemotherapy. 